\title{
Calculation of gas absorption process considering thermal effects
}

\author{
Neumoina N.G. \\ Kamishin technological institute (branch), Volgograd state \\ technical university \\ Kamishin, Russia \\ end@kti.ru
}

Morozova E.V. ${ }^{1,2}$

${ }^{1}$ Kamishin technological institute (branch), Volgograd state technical university

Kamishin, Russia

${ }^{2}$ Saint Petersburg Electrotechnical University "LETI"

Saint Petersburg, Russia end@kti.ru

\begin{abstract}
The absorption process in the petrochemical industry is used to separate, purify and dehydration of hydrocarbon gases. Absorption is the process of absorbing substances from a gas compound with fluids. The absorption process is accompanied by rather significant thermal effects. In this paper, modeling and approbation of the kinetic method for calculating multicomponent gas absorption taking into account the thermal effects of the absorption process, based on the nonlocal version of thermodynamics, are considered. The proposed model provides the complete and consistent accounting for the thermal effects of gas absorption, and this will allow further study of their effect on the accuracy of determining the concentrations of components in the gas and fluid phases with a change in the consumption parameters of the contacting phases. Comparison of the results of calculations based on the proposed model with experimental data for different absorption cases shows its good operability and the possibility of practical application.
\end{abstract}

Keywords—gas absorption; thermal effects; absorbent; heat and mass transfer

\section{INTRODUCTION}

The absorption process in the petrochemical industry is used to separate, purify and dehydrate hydrocarbon gases. Ethane, propane, butane and gasoline components are extracted from natural and associated hydrocarbon gases. With the help of absorption, pyrolysis gases and catalytic cracking gases are also separated and sanitary purification of gases from harmful impurities. [1]

A significant increase in the scale of production with increased attention to environmental issues and the quality of finished products requires further development of scientifically sound and reliable methods for calculating absorption equipment. At present, the widespread use of computer technology and modern software packages makes it possible to use the developed methods for calculating the

\author{
Belov A.V. \\ Kamishin technological institute (branch), Volgograd state \\ technical university \\ Kamishin, Russia \\ end@kti.ru
}

Polivanov A.A.

Kamishin technological institute (branch), Volgograd state technical university

Kamishin, Russia

end@kti.ru

processes and apparatuses of chemical technology in the automated design systems for apparatus of chemical engineering.

The article focuses on the method for calculating the gas absorption, taking into account the thermal effects accompanying this process. Thermal effects are manifested not only in the release of heat during the dissolution of the gas, but also in significant heat transfer between phases and in the transfer of latent heat of vaporescence or condensation of the solvent. The main influence of heat release on the absorption process is to reduce the equilibrium concentration of absorbed gas components on the surface of the absorbent, which reduces the driving force of the mass transfer process.

\section{THE PROBLEM'S STATEMENT}

A number of authors [1-12] carried out studies devoted to the identification of the contribution of thermal effects of gas absorption, the need to take into account these effects when creating of a mathematical model of the process. Experimental studies conducted in stationary and non-stationary conditions confirm the necessity of taking into account thermal effects in gas absorption.

The question arises: on what theoretical basis can we get the most complete description of the joint heat and mass transfer during gas absorption?

At present, it is possible to take into account the influence of thermal effects on mass transfer during absorption in the framework of the kinetic method of describing the process.

But one of the main reasons limiting the use of kinetic methods for calculating processes and apparatus of chemical technology is the lack of reliable methods for determining the mass output coefficients and mass transfer coefficients for multicomponents systems. In addition, within the existing methods of nonisothermal absorption, it is not always possible 
to fully take into account the effect of thermal effects on mass transfer.

The models obtained on the basis of stationary and nonstationary equations of convective diffusion and thermal conductivity [6-10] do not take into account the heat transfer between the contacting phases and the additional fluxes of mass arising due to the temperature difference (thermal diffusion constituent).

The most complete description of heat and mass transfer processes can be obtained if the mass transfer equation is derived from the Stefan-Maxwell equations written for nonisothermal conditions, and the heat transfer process is described by the heat balance equation for the boundary volume with allowance for thermal effects [10].

Of particular importance is the development of methods of calculating for mass-exchange equipment, which require less quantity of priori information and does not rely on any modelmechanism representation.

\section{METHODOLOGY}

Such possibilities for describing the kinetics of heat and mass transfer processes for nonisothermal absorption are discovered within the framework of the nonlocal version of thermodynamics developed by V.P. Maikov [2]. The basis for the non-local version of thermodynamics is the principle of discretization of thermodynamic parameters. This principle allows us to introduce the time parameter in describing thermodynamic equilibrium and treat this state as dynamic and moving. In this case, a transition to the description of nonequilibrium heat and mass transfer processes is simplified significantly.

In order to describe the joint heat and mass transfer during gas absorption based on the nonlocal version of thermodynamics, it is necessary to obtain a mass transfer equation for nonisothermal conditions and an equation for the thermal balance at the phase boundary in which the thermal effects of gas absorption will be taken into account.

Consider the derivation of the mass transfer equation for non-isothermal conditions. The initial expression for this derivation is the equation of flux density for the i-th component under equilibrium conditions:

$$
J_{i}^{*}=c_{S} \cdot \rho \cdot y_{i}
$$

where $c_{S}$ is the speed of sound in a given medium, $\mathrm{m} / \mathrm{s} ; \rho$ is mole density of the compound, $\mathrm{kmol} / \mathrm{m}^{3} ; y_{i}$ is the mole fraction of the component.

Let us now describe the process of transferring mass and heat from one phase to another. When joint heat and mass transfer through the same surface, it is customary to assume that the temperature and concentration differences mainly occur in a thin layer adjacent to the interphase boundary [12]. To describe the nonequilibrium processes occurring in the boundary laminar layer, as a rule, one can apply the linear approximation. Then we write the resultant nonequilibrium density of the mass flux in the linear approximation for nonisothermal conditions in the form:

$$
J_{i}=\Delta J_{i}^{*}=\frac{\partial J_{i}^{*}}{\partial y} \Delta y_{i}+\frac{\partial J_{i}^{*}}{\partial T} \Delta T
$$

Let us estimate the magnitude of elementary difference of concentrations $\Delta \mathrm{y}_{\mathrm{i}}$ and elementary temperature gradient $\Delta \mathrm{T}$, which occurs at a length equal to the characteristic interaction radius $\Delta l$ (Fig. 1).

If in the entire laminar layer thickness $\delta_{y}$ is observed concentration difference $\left(\mathrm{y}_{\mathrm{i}}-\mathrm{y}_{\mathrm{i}}{ }^{*}\right)$ and temperature difference $\left(\mathrm{T}_{\mathrm{y}}-\mathrm{T}^{*}\right)$, where $\mathrm{y}_{\mathrm{i}}^{*}$ and $\mathrm{T}^{*}$ are the equilibrium values of concentrations and temperatures at the boundary of phases, then:

$$
\Delta y_{i}=\frac{y_{i}-y_{i}^{*}}{\delta_{y} / \Delta \ell}
$$

$$
\Delta T=\frac{T_{y}-T^{*}}{\delta_{y} / \Delta \ell} .
$$

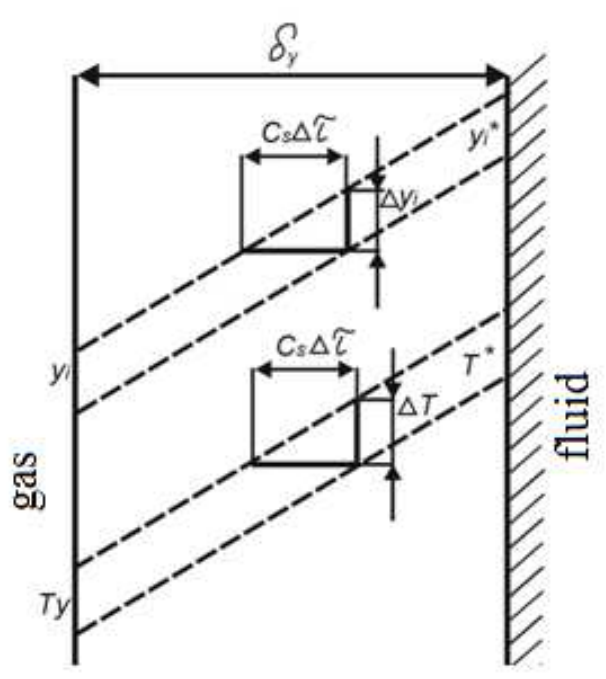

Fig. 1. Conditions on the interphase boundary

Substituting the expressions (for the equilibrium mole flow (1) into (2) and using (3) and (4), we obtain the equation for the nonequilibrium mole flow in nonisothermal conditions:

$$
\begin{aligned}
& J_{y i}=\left(c_{S y} \rho_{y} \frac{\partial y_{i}}{\partial y_{i}}+\rho_{y} y_{i} \frac{\partial c_{S y}}{\partial y_{i}}+c_{S y} y_{i} \frac{\partial \rho_{y}}{\partial y_{i}}\right) \frac{c_{S y} \Delta \tau}{\delta_{y}}\left(y_{i}-y_{i}^{*}\right)+ \\
& +\left(\rho_{y} y_{i} \frac{\partial c_{S y}}{\partial T}+c_{S y} y_{i} \frac{\partial \rho_{y}}{\partial T}\right) \frac{c_{S y} \Delta \tau}{\delta_{y}}\left(T_{y}-T^{*}\right)
\end{aligned}
$$

We represent the mole flux in the form of a product of the flux density and a certain transfer velocity, which we will call the rate of transfer of the substance: 


$$
J_{y i}=c_{C y}\left(\varphi_{M y i}\left(y_{i}-y_{i}^{*}\right)+\varphi_{T y i}\left(T_{y}-T^{*}\right)\right),
$$

where:

$$
\begin{gathered}
c_{C y}=c_{S y}^{2} \Delta \tau / \delta_{y} \\
\varphi_{M y i}=\rho_{y}+\frac{\rho_{y} y_{i}}{c_{S y}} \cdot \frac{\partial c_{S y}}{\partial y_{i}}+y_{i} \frac{\partial \rho_{y}}{\partial y_{i}} \\
\varphi_{T y i}=\frac{\rho_{y} y_{i}}{c_{S y}} \cdot \frac{\partial c_{S y}}{\partial T}+y_{i} \frac{\partial \rho_{y}}{\partial T} .
\end{gathered}
$$

Equations (8) and (9) take into account the dependence of the sound velocity in the medium and the density of the medium (gas or fluid) on the composition and temperature, which corresponds to the physics of the process.

It is convenient to introduce a transfer coefficient $v_{y}$, a dimensionless characteristic of the mass transfer process, instead of the rate of transfer of the substance $c_{C y}$. It is defined by formula:

$$
v_{y}=c_{C y} / c_{S y} .
$$

As a result, we write down the formula for the nonequilibrium mole flow from the side of the gas phase as follows

$$
J_{y i}=v_{y} c_{S y}\left(\varphi_{y i}\left(y_{i}-y_{i}^{*}\right)+\varphi_{T y i}\left(T_{y}-T^{*}\right)\right)
$$

Reasoning in a similar way, one can obtain an expression for the mass flux from the interface of phases to the core of the fluid phase.

It is necessary to draw up an equation for the heat balance for a complete description of the processes of heat and mass transfer.

It is important to take into account all types of thermal effects of gas absorption.

On the basis of the literature review, we distinguish the following types of thermal effects:

1. increase in the temperature of the fluid due to the heat of absorption;

2. lowering the temperature of the fluid due to evaporation of the solvent;

3. heat transfer between the contacting phases;

4. heat transfer between the material flows and the walls of the apparatus (thermal losses).

In the subsequent discussion we shall consider the case of adiabatic absorption (we neglect thermal losses).
We obtain an equation for calculating the heat transfer between phases on the basis of a nonlocal version of thermodynamics.

Для равновесного теплового потока в рамках этой теории можно получить следующее выражение

$$
J_{T}^{*}=c_{S}^{3} \rho_{M},
$$

where $\rho_{M}$ is the mass density of the compound, $\mathrm{kg} / \mathrm{m}^{3}$.

In order to obtain a nonequilibrium heat flux, we again use the linear approximation, we write:

$$
J_{T y}=\Delta J_{T y}^{*}=\frac{d J_{T y}^{*}}{d T} \Delta T .
$$

The value $\Delta \mathrm{T}$ is an elementary temperature gradient, which is determined by equation (4). After differentiation the equilibrium heat flux (12) and substituting equation (4) into (13), we obtain:

$$
J_{T y}=\left(3 c_{S y}^{2} \rho_{M y} \frac{\partial c_{S y}}{\partial T}+c_{S y}^{3} \frac{\partial \rho_{M y}}{\partial T}\right) \frac{c_{S y} \Delta \tau}{\delta y}\left(T_{y}-T^{*}\right) .
$$

We represent the heat flux in the form of a product of the rate of transfer of a substance and the density of the heat flux:

$$
J_{T y}=c_{C y}\left(3 c_{S y} \rho_{M y} \frac{\partial c_{S y}}{\partial T}+c_{S y}^{2} \frac{\partial \rho_{M y}}{\partial T}\right)\left(T_{y}-T^{*}\right) .
$$

The rate of transfer of a substance $\mathrm{c}_{\mathrm{Cy}}$ is determined by the expression (7).

We write the expression for the nonequilibrium heat flow from the side of gas phase:

$$
J_{T y}=\alpha_{y}\left(T_{y}-T^{*}\right)
$$

where:

$$
\begin{gathered}
\alpha_{y}=v_{y} c_{S y} \eta_{T y} \\
\eta_{T y}=3 c_{S y} \rho_{M y} \frac{\partial c_{S y}}{\partial T}+c_{S y}^{2} \frac{\partial \rho_{M y}}{\partial T}
\end{gathered}
$$

where $\alpha_{\mathrm{y}}$ is the heat transfer coefficient in the gas phase, $\mathrm{W} / \mathrm{m}^{2}$; the dimensionless transfer coefficient $v_{y}$ is calculated in (10).

By analogous reasoning, one can obtain an equation for the nonequilibrium heat flux in the fluid phase.

Let us consider the thermal effects of gas absorption. It is usually assumed that when dissolving a gas, heat is released at the phase boundary (similarly, when the solvent is evaporated, 
the phase boundary is cooled). We accept this hypothesis as a working one.

Then the heat balance equation for the selected volume at the phase boundary will be written in the form:

$$
J_{T y}+J_{1}-J_{2}-J_{T x}=0,
$$

where $J_{T y}$ is the heat flux from the core of the gas phase to the interface of phase, $\mathrm{W} / \mathrm{m}^{2} ; J_{T x}$ is heat flow from the interface of phase to the core of the fluid phase, W/ $\mathrm{m}^{2} ; J_{1}$ is absorption heat, $\mathrm{W} / \mathrm{m}^{2} ; J_{2}$ is heat of evaporation of the solvent, $\mathrm{W} / \mathrm{m}^{2}$.

The heat of absorption and the heat of evaporation of the solvent are determined as follows

$$
\begin{aligned}
& J_{1}=\sum_{i=1}^{m} q_{i} J_{i} \\
& J_{2}=r J_{M p}
\end{aligned}
$$

where $\mathrm{q}_{\mathrm{i}}$ is the specific heat of absorption of the i-th component, $\mathrm{J} / \mathrm{kmol} ; \quad r$ is the specific heat of solvent evaporation, $\mathrm{J} / \mathrm{kmol} ; J_{i}$ is the specific mol flux of the i-th component, $\mathrm{kmol} /\left(\mathrm{m}^{2} \mathrm{~s}\right) ; J_{M p}$ is the specific mol flux of solvent, $\mathrm{kmol} /\left(\mathrm{m}^{2} \mathrm{~s}\right)$.

The phase equilibrium condition has the form:

$$
y_{i}^{*}=k_{i} x_{i}^{*}
$$

where, $k_{i}$ is the phase equilibrium constant for the i-th component.

The equation of mass transfer in the gas phase (11), the analogous expression for the nonequilibrium mole flow in the liquid phase, the phase equilibrium condition (22) and the heat irradiation equation for the gas phase (16) and fluid phase form the initial system of equations for describing heat and mass transfer processes for nonisothermal absorption.

Solving this system with respect to the unknowns $y_{i}{ }^{*}, x_{i}{ }^{*}$, $T^{*}, J_{i}, J_{T}$, we get:

1. The mass-transfer equation for nonisothermal conditions:

$$
J_{i}=k_{F i}\left(y_{i}-k_{i} x_{i}+N_{i}\right)
$$

where:

$$
\begin{aligned}
& \frac{1}{k_{F i}}=\frac{1}{\beta_{y i}}+\frac{k_{i}}{\beta_{x i}}, \\
& \beta_{y i}=v_{y} c_{S y} \varphi_{M y i}, \\
& \beta_{x i}=v_{x} c_{S x} \varphi_{M x i},
\end{aligned}
$$

$$
N_{i}=\varphi_{T y i} / \varphi_{M y i}\left(T_{y}-T^{*}\right)+k_{i} \varphi_{T x i} / \varphi_{M x i}\left(T^{*}-T_{x}\right),
$$

$k_{F i}$ is mass transfer coefficient of i-th component, $\mathrm{kmol} /\left(\mathrm{m}^{2} \mathrm{~s}\right) ; \beta_{\mathrm{yi}}, \beta_{\mathrm{xi}}$ is mass delivery coefficients, $\mathrm{kmol} /\left(\mathrm{m}^{2} \mathrm{~s}\right)$.

2. The heat transfer equation:

$$
J_{T y}=\frac{1}{1 / \alpha_{y}+1 / \alpha_{x}}\left(T_{y}-T_{x}\right)+\frac{1}{1+\alpha_{y} / \alpha_{x}}\left(J_{1}-J_{2}\right) .
$$

The heat flux in the liquid phase is determined from the balance ratio (19).

The equation for calculating the equilibrium temperature at the interface of phase:

$$
T^{*}=\frac{\alpha_{y} T_{y}+J_{1}-J_{2}+\alpha_{x} T_{x}}{\alpha_{y}+\alpha_{x}} .
$$

The quantities $\varphi_{\mathrm{Myi}}\left(\varphi_{\mathrm{Mxi}}\right)$ in (25) - (27) and $\eta_{T y}\left(\eta_{T y}\right)$ in (17), (18) are calculated theoretically in (8). They depend on the physical properties of the compound: the speed of sound, density, molecular weight.

The dimensionless transfer coefficient of a substance characterizes the hydrodynamic conditions $v_{y}\left(v_{x}\right)$ in which heat and mass transfer processes occur. In general, the transfer coefficient is less than unity.

In the limit, it can be equal to unity, and then $\mathrm{c}_{\mathrm{Cy}}=\mathrm{c}_{\mathrm{Sy}}$, $\delta_{\mathrm{y}}=\Delta l$, that is, the process of mass and heat transfer will occur with the maximum possible speed equal to the speed of sound in a given medium, and the thickness of the boundary laminar layer $\delta_{\mathrm{y}}$ will be equal to the characteristic linear dimension $\Delta l$.

To verify the adequacy of the proposed model, an algorithm was compiled and the countercurrent absorber was calculated.

\section{THE RESULTS OF STUDIES}

The calculation was made for various options of absorber: falling-film absorber, packed absorber and plate absorber in the verification version of the calculation.

The model is insensitive to the design of the apparatus, since the hydrodynamic situation at the interface of phases corresponds to the transport coefficient $v_{y}\left(v_{x}\right)$ in (10), which is selected during the calculation.

For falling-film absorber, the transport coefficient in the fluid phase $v_{x}$ was determined on the basis of the assumption that the concentration change occurs $\left(x_{i}^{*}-x_{i}\right)$ over the entire thickness of the fluid film (the thickness of the boundary laminar layer in the fluid phase is equal to the thickness of the film of fluid). The transport coefficient in the gas phase $v_{y}$ was selected during the calculation to the best agreement between the experimental and calculated data. 
Table 1 shows the values of the transport coefficient in the gas phase $v_{y}$ and the corresponding thicknesses of the diffusion layer $\delta_{\mathrm{y}}$ in the gas, depending on the gas velocity $\mathrm{W}$ in the pillar for two different irrigation density $\mathrm{q}$.

TABLE I. THE VALUES OF THE TRANSFER COEFFICIENT AND THE THICKNESS OF THE DIFFUSION LAYER IN THE GAS PHASE OF A FALLING-FILM ABSORBER

\begin{tabular}{|c|c|c|c|}
\hline \multirow{2}{*}{ Indices } & \multicolumn{3}{|c|}{ Gas velocity $W, \mathrm{~m} / \mathrm{s}$} \\
\hline & 1.3 & 3.0 & 5.3 \\
\hline \multicolumn{4}{|c|}{$\mathrm{q}=22.3 \cdot 10^{-6}, \mathrm{~m}^{3} /(\mathrm{m} \cdot \mathrm{s})$} \\
\hline$v_{y}$ & $3.3 \cdot 10^{-5}$ & $5.3 \cdot 10^{-5}$ & $8.0 \cdot 10^{-5}$ \\
\hline$\delta_{\mathrm{y}}$ & $1.3 \cdot 10^{-7}$ & $0.8 \cdot 10^{-7}$ & $0.5 \cdot 10^{-7}$ \\
\hline \multicolumn{4}{|c|}{$\mathrm{q}=70.0 \cdot 10^{-6}, \mathrm{~m}^{3} /(\mathrm{m} \cdot \mathrm{s})$} \\
\hline$v_{y}$ & $3.3 \cdot 10^{-5}$ & $7.4 \cdot 10^{-5}$ & $11.8 \cdot 10^{-5}$ \\
\hline$\delta_{\mathrm{y}}$ & $1.3 \cdot 10^{-7}$ & $0.6 \cdot 10^{-7}$ & $0.4 \cdot 10^{-7}$ \\
\hline
\end{tabular}

Analysis of the tabular data shows that with increasing gas velocity in the pillar, the thickness of the diffusion layer in the gas phase decreases, and the transport coefficient increases. This is in good agreement with the physical meaning that is embedded in the transport coefficient $v_{y}$.

Calculation of a backflow plate absorber for the separation of a multicomponent hydrocarbon compound was also performed. As an absorbent, a kerosene fraction with a molecular weight of $\mathrm{M}=210 \mathrm{~kg} / \mathrm{kmol}$ and density of $\rho_{\mathrm{x}}=820 \mathrm{~kg} / \mathrm{m}^{3}$ was used. The amount of gas supplied to the absorption is $G_{n+1}=0.514 \mathrm{kmol} / \mathrm{s}$, the amount of lean absorbent is $L_{0}=0.182 \mathrm{kmol} / \mathrm{s}$, the average temperature of the fluid is $T_{x}=307 \mathrm{~K}$. The transport coefficients for the gas phase is $v_{y}=2 \cdot 10^{-6}$ and transport coefficients for the fluid phase is $v_{x}=8.3 \cdot 10^{-8}$, the working height of the apparatus is $\mathrm{H}=3.3 \mathrm{~m}$, the average gas temperature is $\mathrm{T}_{\mathrm{y}}=308 \mathrm{~K}$, the specific contact area of the phases per unit volume is $\sigma=200 \mathrm{~m}^{2} / \mathrm{m}^{3}$.

The experimental and calculated values of the component concentrations in the inlet and outlet streams of the plate absorber are shown in Table 2. Experimental data are taken from [13].

TABLE II. EXPERIMENTAL AND CALCULATED VALUES OF CONCENTRATIONS (MULTICOMPONENT HYDROCARBON MIXTURE)

\begin{tabular}{|c|c|c|c|c|c|c|c|c|}
\hline \multirow[b]{2}{*}{$\begin{array}{l}\text { component of } \\
\text { absorption }\end{array}$} & \multicolumn{8}{|c|}{ Component of mixture } \\
\hline & $\mathrm{N}_{2}$ & $C_{1}$ & $C_{2}$ & $C_{3}$ & $i-C_{4}$ & $n-C_{4}$ & $C_{5}$ & $\dot{\vec{\Xi}}$ \\
\hline $\begin{array}{l}\text { crude gas } \\
\text { (experiment) }\end{array}$ & 0.110 & 0.364 & 0.211 & 0.215 & 0.036 & 0.037 & 0.016 & 0.000 \\
\hline $\begin{array}{l}\text { skinny } \\
\text { absorbent } \\
\text { (experiment) }\end{array}$ & 0.000 & 0.000 & 0.000 & 0.015 & 0.010 & 0.042 & 0.022 & 0.911 \\
\hline $\begin{array}{l}\text { dry gas } \\
\text { (experiment) }\end{array}$ & 0.151 & 0.477 & 0.240 & 0.112 & 0.006 & 0.010 & 0.004 & - \\
\hline $\begin{array}{l}\text { dry gas } \\
\text { (design value) }\end{array}$ & 0.132 & 0.422 & 0.218 & 0.171 & 0.021 & 0.030 & 0.007 & - \\
\hline $\begin{array}{l}\text { Saturated } \\
\text { absorbent }\end{array}$ & 0.000 & 0.007 & 0.053 & 0.192 & 0.041 & 0.113 & 0.039 & 0.555 \\
\hline
\end{tabular}

\begin{tabular}{|c|c|c|c|c|c|c|c|c|}
\hline \multirow[b]{2}{*}{$\begin{array}{c}\text { component of } \\
\text { absorption }\end{array}$} & \multicolumn{8}{|c|}{ Component of mixture } \\
\hline & $\mathrm{N}_{2}$ & $C_{1}$ & $C_{2}$ & $C_{3}$ & $i-C_{4}$ & $n-C_{4}$ & $C_{5}$ & 䓂 \\
\hline \multicolumn{9}{|l|}{ (experiment) } \\
\hline $\begin{array}{l}\text { Saturated } \\
\text { absorbent } \\
\text { (design value) }\end{array}$ & 0.002 & 0.030 & 0.059 & 0.151 & 0.042 & 0.071 & 0.035 & 0.610 \\
\hline
\end{tabular}

Comparison of the experimental and calculated values of the outlet concentrations in a dry gas and a saturated absorbent shows good agreement between them.

\section{CONCLUSIONS}

Summing up, it can be concluded that the proposed kinetic method for calculating multicomponent nonisothermal gas absorption, developed within the framework of the non-local version of thermodynamics, allows us to disclose the physical meaning of coefficients: mass transfer coefficient, mass delivery coefficient, heat irradiation coefficient and heat transfer coefficient. It allows us to denote the hydrodynamic component of these coefficients.

In addition, the proposed model provides the complete and consistent accounting for the thermal effects of gas absorption, and this will allow further study of their effect on the accuracy of determining the concentrations of components in the gas and fluid phases with a change in the consumption parameters of the contacting phases.

Comparison of the results of calculations based on the proposed model with experimental data for different absorption cases shows its good operability and the possibility of practical application.

\section{References}

[1] A.I. Scoblo, Yu.A. Molokanov, A.I. Vladimirov and V.A. Shchelkunov, Processes and apparatuses of oil and gas processing and petrochemistry. Moscow, IC of the Gubkin Russian State University of Oil and Gas, 2012. 725p.

[2] N.G. Neumoina, A.V. Belov, "Creation of model of the kinetic method for calculating multicomponent nonisothermal absorption of gases", J. Modern problems of science and education, Vol 6, 2014. http://scienceeducation.ru/ru/article/view?id=15421. Accessed 01.03.2018.

[3] N.G. Neumoina, A.V. Belov, "Approbation of model of the kinetic method of calculation to multicomponent nonisothermal absorption of gases", J. Modern problems of science and education, Vol. 6, 2014. https://science-education.ru/pdf/2014/6/149.pdf. Accessed 01.03.2018.

[4] J.R. Born, U. Stockar, and G.C. Coggan, "Gas absorption with heat effects. 1. A new computational method", J. Ind. Eng. Chem. Process Des. Develop., vol. 13, No 2, 1974, pp.115-123.

[5] E.Ya. Koenig, LP Kholpanov, and L.I. Katysheva, "Calculation of twophase nonisothermal absorption in a liquid film in the mode of downward direct flow. J. TOXT, vol 19, No 2, pp.147-152.

[6] A.S. Zavorin, G.V. Kuznetsov, "The contribution of the scientific school of academician V.E. Nakoryakov in thermophysics and heat engineering", J. Proceedings of Tomsk Polytechnic University. Engineering georesources. Vol.326, No. 7, 2015, pp. 130-137.

[7] S.H. Chiang, H.L. Toor, "Gas absorption accompanied by large heat effects and volume change of liquid phase", J. AIChE Journale, vol. 10, No 3, 1964, pp. 398-402.

[8] S.L. Verma, G.B. Delencey, "Thermal effects in gas absorption", J. AIChE Journale, vol. .21, No 1, 1975, pp. 96-102. 
[9] A.K.. Surech, S. Asai, and O.E. Potter, "Temperature-dependent physical properties in physical gas absorption", J. Chem. Eng. Science, vol. 38, No 1, 1983, pp.127-133

[10] Kh.E. Gorand, I.A. Kamenev, and Yu.I. Kallas, "Conjugated heat and mass transfer in a multicomponent vapor-liquid system", J. JACh, vol. 58 , No 2, 1985, pp.450-452.
[11] T. Sherwood, R. Pigford, and Ch. Wilke, Masstransfer , Moscow: Chemistry, 1982.696 p.

[12] R.G. Galeeva, Investigation of the absorption of petroleum (associated) gases. Cand. Diss. Moscow, 1970, 143 p.

[13] V.P Maikov, The extended version of classic thermodynamics is physics of discrete space-time. Moscow: MGUIE, 1997, $160 \mathrm{p}$. 